\title{
Original
}

\section{Linfoma testicular primario: experiencia en el Instituto Nacional de Enfermedades Neoplásicas. Lima-Perú}

\author{
Gilmer A. Díaz Pérez, Mariela Pow-Sang Godoy, Carlos Morante Deza, Luis Meza Montoya, \\ Víctor Destefano Urrutia
}

Urología Oncológica del Hospital General Santa Rosa. Instituto Nacional de Enfermedades Neoplásicas "Dr. Eduardo Cáceres Graziani”. Lima, Perú

\section{Resumen}

Introducción: El linfoma testicular es una enfermedad poco frecuente con características peculiares y un pronóstico pobre. Material y métodos: Evaluamos en forma retrospectiva 32 pacientes con este diagnóstico estudiando sus características epidemiológicas, valores hematológicos, tipo histológico, lugares de metástasis, tratamiento recibido y tiempo de sobrevida. Asímismo comparamos nuestros resultados con los reportes internacionales, haciendo énfasis en la necesidad de estudios prospectivos para obtener mejores conclusiones.

Resultados: El promedio de edad fue de 45 años, con más de la mitad de los pacientes con estadío clínico IV al momento del diagnóstico, con la variedad histiocítica como la patología más frecuente, y con el tiempo promedio de sobrevida de $39,543 \pm 14,451$ meses, y el tiempo mediano (tiempo en el cual muere el 50\% de pacientes) de 15 $\pm 7,025$ meses.

Conclusiones: Enfermedad poco frecuente cuyo pronóstico es aún pobre, con una sobrevida promedio de 40 meses y tiempo mediano de 15 meses.

Palabras clave: Linfoma testicular.

Primary testicular lymphoma: experience Instituto especializado de enfermedades neoplásicas. Lima-Peru

\section{Abstract}

Introduction: Testicular lymphoma is a rare illness with peculiar characteristics but with a poor prognosis.

Material and methods: We evaluated 32 patients retrospectively studying their epidemiologic characteristics, hematologic values, histologic type, metastasis sites, the treatment given and the survival. We compared our results with international reports and we think that prospective studies are needed for better conclusions.

Results: The average of age was 45 years old, with more than the half of patients with clinical stage IV at the moment of the diagnosis, the histiocitic pathology was the most frequent, and the time of survival was 39,543 $\pm 14,451$ months and the time in which the $50 \%$ of the patients die is $15 \pm 7,025$ months.

Conclusions: This is a rare disease, with a very poor prognosis, with a time of survival of $39,543 \pm 14,451$ months and the time in which the $50 \%$ of the patients die is $15 \pm 7,025$ months.

Keywords: Testicular lymphoma.

$\mathrm{E}^{1}$ Linfoma No-Hodgkin que se origina en el testículo es una enfermedad rara, representa sólo el $1 \%$ de todos los linfomas ${ }^{1}$ con una incidencia de 0,26/100.000 por año y el 5\% de todas las neoplasias testiculares ${ }^{2}$. El Linfoma explica el $2 \%$ de los tumores testiculares en varones jóvenes pero el 25\% en varones mayores ${ }^{3,4}$ y representa el $50 \%$ de los tumores testiculares en mayores de 50 años de edad $^{5}$. Debido a que este tumor es relativamente raro hay pocas series que documentan su evolución y lo encontrado en la literatura sugiere un pobre pronóstico de los linfomas extranodales sea cual fuere su ubicación en el cuerpo ${ }^{6,7}$. La orquidectomía es el tratamiento inicial estándar ante la sospecha de un tumor testicular maligno. Según la literatura el $80-90 \%$ son de células B grandes difuso ${ }^{5}$ pero casos aislados de otros subtipos histológicos han sido descritos ${ }^{8-10}$. Los sitios a los que puede invadir son múltiples ${ }^{11-13}$, siendo la diseminación sistémica frecuente. A través de los años, el tratamiento ha sido variable, la radioterapia sin lograr un control óptimo ${ }^{14}$, la quimioterapia en diversos esquemas $^{12,13}$, siendo terapia recomendada después de la orquidectomía $^{15}$. El pronóstico es aún pobre con un alto porcentaje de recurrencia ${ }^{12,16}$. Es objetivo de este trabajo revisar los casos de linfoma testicular 
primario de nuestra institución ya que se trata de una patología poco frecuente, describir algunas de sus características y evaluar su pronóstico mediante datos de sobrevida de los pacientes.

\section{MATERIAL Y MÉTODOS}

El Departamento de Estadística de nuestra institución realizó una búsqueda de todos los casos de linfoma testicular primario desde el año 1978 hasta el año 2005.

Encontramos 32 historias clínicas que fueron revisadas para determinar las características de los pacientes en lo que respecta a edad, valores hematológicos al diagnóstico, lado de la tumoración, tipo histológico de la misma, tratamiento recibido, lugares de metástasis y sobrevida.

Todos los resultados de patología fueron informados por patólogos de nuestra institución.

Para encontrar la sobrevida se tomaron como parámetros la fecha de admisión al hospital y la fecha de muerte cuando ésta estuvo consignada en la historia clínica o la fecha del último control del paciente cuando éste se encontraba vivo. En los casos de pacientes perdidos de vista revisamos el estado de la enfermedad según el último control reportado en la historia clínica, ya sea con evidencia de enfermedad, en progresión de enfermedad, sin evidencia de enfermedad y desconocido.

Los datos fueron tabulados en forma simple, en caso de las variables continuas determinamos la media y la desviación estándar, y en las variables categóricas determinamos los porcentajes respectivos.

Se calculó la sobrevida con el método de KaplanMeier con la fecha de diagnóstico como fecha inicial. La longitud de la sobrevida fue calculada hasta la fecha del último dato de seguimiento o la fecha de muerte.

\section{RESULTADOS}

Encontramos 32 pacientes cuyas edad promedio fue de 49,53 $\pm 20,03$ años (1-75), la hemoglobina tuvo un promedio de $120,6 \pm 18,01 \mathrm{mg} / \mathrm{dL}$ (80-150), el número de plaquetas fue de $347 \pm 145,59 \times 10^{9}(85-$

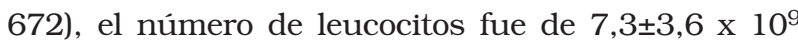
(1,1-17,45), la deshidrogenada láctica (DHL) fue de $437,06 \pm 475,72$ U/L (55-1800) y el tiempo de sobrevida fue en promedio 29 $\pm 50,97$ meses (1-164). Es importante recalcar que el diagnóstico fue histológico después de orquidectomía radical en todos los casos.
El lado mayormente afectado fue el izquierdo con un 50\%, mientras que el lado derecho se vio afectado en un 43,75\% y ambos testículos, en forma sin crónica, en un $6,25 \%$. El porcentaje según estadío fue como sigue: I (15,63\%), II (12,5\%), III $(12,5 \%) \mathrm{y}$ IV $(53,13 \%)$, mientras que en el $6,25 \%$ se desconoce el estadío por datos insuficientes en la historia clínica. La infiltración de la médula ósea estuvo presente en el 6,25\% de los pacientes. No se encontraron metástasis retroperitoneales en el 37,5\% de los pacientes, se encontraron metástasis retroperitoneales menores de $2 \mathrm{~cm}$ en el 3,13\%, entre 2 a $5 \mathrm{~cm}$. en el $15,63 \%$ y mayores de $5 \mathrm{~cm}$ en el $31,25 \%$, mientras que en el restante $12,5 \%$ no se encontraron datos suficientes al respecto. El tipo histológico más frecuente fue el histiocítico con un 46,88\%, luego el linfocítico con un $21,88 \%$, seguido por el de células B grandes difuso con un 18,75\%, células del manto con $6,25 \%$ y finalmente células tipo Burkitt y $\mathrm{T}$ periféricas con $3,13 \%$ cada uno.

Los lugares donde se encontraron metástasis fueron: hueso, sistema nervioso central, pericardio, pulmón, globo ocular, mandíbula, glándula suprarrenal, bazo, piel, conjuntiva y partes blandas.

El tratamiento que recibieron fue con quimioterapia en 40,63\% de los pacientes, quimio y radioterapia en el 34,38\% y sólo radioterapia en el 9,38\% de ellos; 5 pacientes que corresponden al 15,63\% de los casos no recibieron ningún tipo de tratamiento porque no acudieron a los controles en las citas programadas, es decir se perdieron de vista.

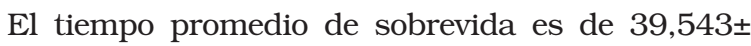
14,451 meses, y el tiempo mediano (tiempo en el cual muere el 50\% de pacientes) de 15 $\pm 7,025$ meses. Estos datos de sobrevida se pueden ver reflejados en la Tabla 1 (Tabla de Supervivencia) y Tabla 2 (Medias y Medianas del Tiempo de Supervivencia) y en la Figura 1 (Función de Supervivencia).

\section{DISCUSIÓN}

No hay estudios prospectivos de linfoma testicular primario debido a que se trata de una patología poco frecuente, un peculiar subtipo de linfoma que difiere del linfoma ganglionar en muchos aspectos ${ }^{10}$. Histológicamente el 80-90\% de los linfomas primarios del testículo son de células $\mathrm{B}$ grandes difuso ${ }^{5}$ pero casos aislados de otros subtipos histológicos han sido reportados. Histologías peculiares y características moleculares han sido descritas ${ }^{8-9}$ incluyendo la diferenciación plasmacitoide y la hipermu- 
Tabla 1. Tabla de supervivencia

\begin{tabular}{|c|c|c|c|c|c|c|}
\hline & & & $\begin{array}{l}\text { Proporción acu } \\
\text { hasta el mome }\end{array}$ & que sobrevive & & \\
\hline & Tiempo & Estado & Estimación & Error típico & $\begin{array}{c}\mathbf{N}^{\circ} \text { de eventos } \\
\text { acumulados }\end{array}$ & $\begin{array}{c}\mathrm{N}^{\circ} \text { de casos que } \\
\text { permanecen }\end{array}$ \\
\hline 1 & 1.000 & 1.00 & . & . & 1 & 31 \\
\hline 2 & 1.000 & 1.00 & . & . & 2 & 30 \\
\hline 3 & 1.000 & 1.00 & .906 & .052 & 3 & 29 \\
\hline 4 & 1.000 & .00 & . & . & 3 & 28 \\
\hline 5 & 1.000 & .00 & . & . & 3 & 27 \\
\hline 6 & 1.000 & .00 & . & . & 3 & 26 \\
\hline 7 & 2.000 & 1.00 & . & . & 4 & 25 \\
\hline 8 & 2.000 & 1.00 & . & . & 5 & 24 \\
\hline 9 & 2.000 & 1.00 & .802 & .073 & 6 & 23 \\
\hline 10 & 2.000 & .00 & . & . & 6 & 22 \\
\hline 11 & 2.000 & .00 & . & . & 6 & 21 \\
\hline 12 & 3.000 & 1.00 & .764 & .079 & 7 & 20 \\
\hline 13 & 4.000 & 1.00 & .725 & .084 & 8 & 19 \\
\hline 14 & 5.000 & 1.00 & .687 & .087 & 9 & 18 \\
\hline 15 & 8.000 & 1.00 & . & . & 10 & 17 \\
\hline 16 & 8.000 & 1.00 & .611 & .093 & 11 & 16 \\
\hline 17 & 8.000 & .00 & . & . & 11 & 15 \\
\hline 18 & 10.000 & 1.00 & .570 & .095 & 12 & 14 \\
\hline 19 & 10.000 & .00 & . & . & 12 & 13 \\
\hline 20 & 10.000 & .00 & . & . & 12 & 12 \\
\hline 21 & 10.000 & .00 & . & . & 12 & 11 \\
\hline 22 & 12.000 & 1.00 & .518 & .100 & 13 & 10 \\
\hline 23 & 13.000 & .00 & . & . & 13 & 9 \\
\hline 24 & 14.000 & .00 & . & . & 13 & 8 \\
\hline 25 & 15.000 & 1.00 & .453 & .106 & 14 & 7 \\
\hline 26 & 22.000 & 1.00 & .389 & .109 & 15 & 6 \\
\hline 27 & 26.000 & 1.00 & .324 & .108 & 16 & 5 \\
\hline 28 & 29.000 & 1.00 & .259 & .104 & 17 & 4 \\
\hline 29 & 32.000 & .00 & . & . & 17 & 3 \\
\hline 30 & 33.000 & 1.00 & .173 & .099 & 18 & 2 \\
\hline 31 & 61.000 & .00 & . & . & 18 & 1 \\
\hline 32 & 164.000 & .00 & . & . & 18 & 0 \\
\hline
\end{tabular}

tación somática de la inmunoglobulina de cadena pesada, indicando una posible estimulación antígeno dirigida, análoga a la que se ha visto en el linfoma de la zona marginal extranodal ${ }^{10}$. En nuestra revisión las patologías más frecuentes fueron diferentes a las vistas en la literatura y fueron en orden descendente la histiocítica, la linfocítica y en tercer lugar la de células grandes B difuso. El pobre pronóstico de la mayoría de pacientes ha sido confir- mado también en este estudio con una pequeña proporción de pacientes curados. Incluso para pacientes con estadío I el pronóstico es peor que para aquellos con linfoma en otros sitios. Los resultados del tratamiento sobre la evolución de los pacientes es muy pobre, $\mathrm{y}$ la recurrencia incluso en las estadíos I y II llega hasta un $60 \%{ }^{1}$. La duración de la sobrevida después de la recurrencia parece también ser pobre. Las fallas ocurren usualmente 
Tabla 2. Medias y medianas del tiempo de supervivencia

\begin{tabular}{|c|c|c|c|c|c|c|c|}
\hline \multicolumn{4}{|l|}{ Media $^{(a)}$} & \multicolumn{4}{|l|}{ Mediana } \\
\hline \multirow[b]{2}{*}{ Estimación } & \multirow[b]{2}{*}{ Error típico } & \multicolumn{2}{|c|}{ Intervalo de confianza al $\mathbf{9 5 \%}$} & \multirow[b]{2}{*}{ Estimación } & \multirow[b]{2}{*}{ Error típico } & \multicolumn{2}{|c|}{ Intervalo de confianza al $\mathbf{9 5 \%}$} \\
\hline & & Limite inferior & Limite superior & & & Limite inferior & Límite superior \\
\hline 39.543 & 14.451 & 11.219 & 67.867 & 15.000 & 7.025 & 1.230 & 28.770 \\
\hline
\end{tabular}

(a)La estimación se limita al mayor tiempo de supervivencia si se ha censurado.

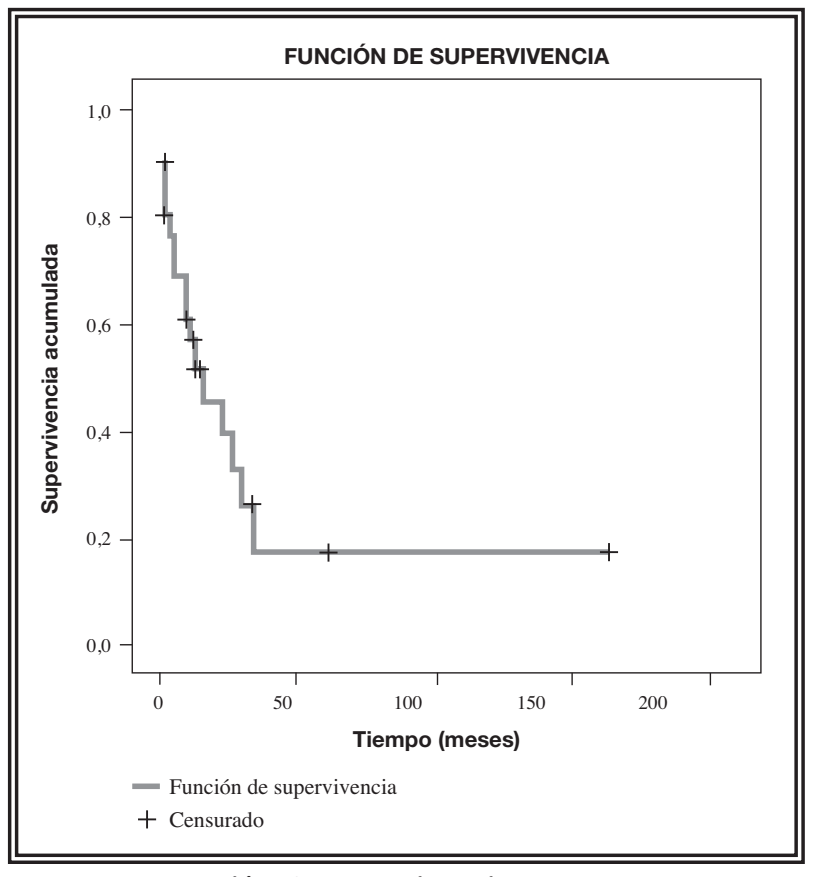

FIGURA 1. Función de supervivencia.

dentro de 1 a 3 años después de la terapia inicial, sin embargo también pueden observarse recurrencias hasta 14 años después del diagnóstico especialmente en el SNC y en el testículo contralateral lo que hace difícil distinguir de una nueva enfermedad primaria de una recurrencia tardía. Esta enfermedad tiene un alto porcentaje de recurrencia nodal cuya causa permanece aún sin definir ${ }^{17}$. Un particular patrón de expresión de moléculas de adhesión, que resulta en una pobre adhesión de las células del linfoma a la matriz extracelular, podría contribuir a esta conducta $^{18-19}$. En suma, tanto el testículo como el SNC han sido considerados como sitios inmunoprivilegiados donde las células de linfoma podrían escapar al inmunoseguimiento mediado por los linfocitos $\mathrm{T}^{20} \mathrm{y}$ han sido considerados como santuarios donde la quimioterapia disminuye su eficacia.

A través de los años, el tratamiento ha sido variable, la radioterapia no logra un control óptimo de la enfermedad incluso para pacientes en estadío ${ }^{14}$, la quimioterapia sin antraciclínicos ha mostrado resultados inferiores comparado a los regimen con antraciclínicos ${ }^{12,13}$, siendo esta la terapia recomendada después de la orquidectomía ${ }^{15}$. Sin embargo, a pesar de la administración de terapia agresiva el pronóstico es aún pobre incluso en la enfermedad localizada con un porcentaje de recurrencia a 2 años que excede el 50\% en la mayoría de las series $^{12,16}$. La quimioterapia adyuvante después de la orquidectomía y la irradiación retroperitoneal fueron introducidas a comienzos de los 80, y algunos reportes mostraron una reducción en las recaídas y mejora en la sobrevida global para los pacientes tratados con quimioterapia ${ }^{21}$. Sin embargo, el beneficio de la quimioterapia agresiva para el linfoma testicular en estadío temprano es controvertido ${ }^{21-23}$. Actualmente la radioterapia retroperitoneal ha sido habitualmente abandonada en los pacientes con estadío $\mathrm{I}^{24}$, pero es generalmente dada a los pacientes con estadío II de acuerdo a las recomendaciones del tratamiento combinado ${ }^{25}$.

Esta es una enfermedad generalmente de adultos mayores que tienen poca tolerancia a los efectos de la quimioterapia, sin embargo en nuestro estudio el promedio de edad fue menor de 50 años en comparación con otras revisiones cuyo promedio de edad es por encima de $60^{26}$. En nuestro estudio encontramos que la mayoría de pacientes tenían un estadío avanzado de la enfermedad, estadío III o IV, a diferencia de otros estudios en donde los estadíos I y II comprenden el $79 \%$ de pacientes ${ }^{26}$. En todos los casos, los marcadores tumorales para tumores de células germinales de origen testicular fueron normales en nuestra serie y la deshidrogenasa láctica (DHL) fluctuó entre 55 a 1.800 U/L con casi la mitad de los pacientes con valores por encima de lo normal a diferencia de otros estudios en donde la mayoría de sus pacientes tienen valores en el rango normal $^{26}$. El linfoma primario testicular muestra una tendencia a diseminarse sistémicamente a múltiples sitios extranodales incluyendo el testículo 
contralateral, el sistema nervioso central, la piel, al anillo de Waldeyer, el pulmón, la pleura y tejidos blandos ${ }^{11-13}$, algunos de los cuales también hemos encontrado en nuestros pacientes.

La sobrevida en estos pacientes es pobre ${ }^{12,16,26}$, algo que también hemos encontrado en nuestro trabajo, en el que la gran mayoría de pacientes tuvo una sobrevida menor de un año, sin embargo encontramos también a dos pacientes con tiempos de sobrevida mayores de 5 años.

\section{CONCLUSIONES}

El linfoma testicular es una enfermedad poco frecuente cuyo pronóstico es aún pobre incluso en estadíos tempranos de la enfermedad y cuyo tiempo promedio de sobrevida es de 39,543 $\pm 14,451$ meses, y el tiempo mediano (tiempo en el cual muere el 50\% de pacientes) de 15 $\pm 7,025$ meses.

\section{Agradecimientos}

Al Sr. Alberto Minchón Medina, quien se encargó del análisis estadístico.

\section{REFERENCIAS}

1. Aisenberg AC. Malignant lymphoma: biology, natural history, and treatment. Philadelphia, PA: Lea, \& Febiger, 1991.

2. Doll DC, Weiss RB. Malignant lymphoma of the testis. Am J Med. 1986;81(3):515-524

3. Eckert H, Smith JP. Malignant lymphoma of the testis. Brit Med J. 1963;2(5362):891-894.

4. Sussman EB, Hajdu SI, Lieberman PH, Whitmore WF. Malignant lymphoma of the testis: a clinicopathologic study of 37 cases. J Urol. 1977;118(6):1004-1007.

5. Moller MB, D Amore F, Christensen BE. Testicular lymphoma: A population-based study of incidence, clinicophatological correlations and prognosis. The Danish Lymphoma Study Group, LYFO. Eur J Cancer. 1994;30A(12):1760-1764.

6. Tepperman BS, Gospodarowicz MK, Bush RS, Brown TC. NonHodgkin's lymphoma of the testis. Radiology, 142: 203, 1982. Internet Resources Bibliographic Links Library Holdings.

7. Doll DC, Weiss RB. Malignant lymphoma of the testis. Amer J Med. 81: 515, 1986. Internet Resources Bibliographic Links Library Holdings.

8. Ferry JA, Harris NL, Young RH, et al. Malignant lymphoma of the testis, epididymis, and spermatic cord: A clinicopathologic study of 69 cases with immunophenotypic analysis. Am J Surg Pathol 18:376-390, 1994.

9. Chan JK, Tsang WY, Lau WH, et al. Aggressive T/natural killer cell lymphoma presenting as testicular tumor. Cancer 77:11981205, 1996.

10. Hyland J, Lasota J, Jasinski M, et al. Molecular pathological analysis of testicular diffuse large cell lymphomas. Hum Pathol 29:1231-1239, 1998.

11. Fonseca R, Habermann TM, Colgan JP, et al. Testicular lymphoma is associated with a high incidence of extranodal recurrence. Cancer 88:154-161, 2000.
12. Crellin AM, Hudson BV, Bennett MH, et al: Non-Hodgkin's lymphoma of the testis. Radiother Oncol 27:99-106, 1993.

13. Martenson JA Jr, Buskirk SJ, Ilstrup DM, et al: Patterns of failure in primary testicular non-Hodgkin's lymphoma. J Clin Oncol 6:297-302, 1988.

14. Jackson SM, Montessori GA: Malignant lymphoma of the testis: Review of 17 cases in British Columbia with survival related to pathological subclassification. J Urol 123:881-883, 1980.

15. Tepperman BS, Gospodarowicz MK, Bush RS, et al: NonHodgkin lymphoma of the testis. Radiology 142:203-208, 1982.

16. Tondini C, Ferreri AJM, Siracusano L, et al: Diffuse large lymphoma of the testis. J Clin Oncol 17:2854-2858, 1999.

17. Visco C, Medeiros LJ, Mesina OM, et al: Non Hodgkin's lymphoma affecting the testis: Is it curable with doxorubicin based therapy? Clin Lymphoma 2:40-46, 2001

18. Drillenburg P, Pals ST. Cell adhesion receptors in lymphoma dissemination. Blood. 2000;95(6):1900-1910.

19. Horstmann WG, Timens W. Lack of adhesion molecules in testicular diffuse centroblastic and immunoblastic B cell lymphomas as a contributory factor in malignant behavior. Virchows Arch. 1996;429(2-3):83-90.

20. Riemersma SA, Jordanova ES, Schop RF, Philippo K, Looijenga LH, Schuuring E, et al. Extensive genetic alterations of the HLA region, including homozygous deletions of HLA class II genes in B-cell lymphomas arising in immune-privileged sites. Blood. 2000;96(10):3569-3577.

21. Roche H, Suc E, Pons A, Woodman F, Huguet-Rigal F, Cave riviere $\mathrm{P}$, et al. Stage IE non Hodgkin's lymphoma of the testis: A need for a brief aggressive chemotherapy. J Urol. 1989;141 (3):554-556.

22. Pectasides D, Economopoulos T, Kouvatseas G, Antoniou A, Zoumbos Z, Aravantinos G, et al. Anthracyclinebased chemotherapy of primary non-Hodgkin's lymphoma of the testis: The Hellenic Cooperative Oncology Group experience. Oncology. 2000;58(4):286-292.

23. Sasai K, Yamabe H, Tsutsui K, Dodo Y, Ishigaki T, Shibamoto Y, et al. Primary testicular non Hodgkin's lymphoma: A clinical study and review of the literature. Am J Clin Oncol. 1997;20(1):59-62.

24. Zietman AL, Coen JJ, Ferry JA, Scully RE, Kaufman DS, McGovern FG. The management and outcome of stage IAE non Hodgkin's lymphoma of the testis. J Urol. 1996;155(3):943946.

25. Miller TP, Dahlberg S, Cassady JR, Adelstein DJ, Spier CM, Grogan TM, et al. Chemotherapy alone compared with chemotherapy plus radiotherapy for localized intermediate and highgrade non Hodgkin's lymphoma. N Engl J Med 1998;339(1):2126.

26. Zucca E, Conconi A, Mughal TI, Sarris AH, Seymour JF, Vitolo U, et al. Patterns of outcome and prognostic factors in primary large-cell lymphoma of the testis in a survey by the International Extranodal Lymphoma Study Group. J Clin Oncol. 2003;21(1):20-27.

Correspondencia autor: Dr. Gilmer A. Díaz Pérez Urología Oncológica del Hospital General Santa Rosa. Av. Bolívar s/n cuadra 8 - Pueblo Libre, Lima 21. Perú Tel.: 511-6158200 - anexo 160

E-mail autor: gilmeruro@yahoo.com/gilmerqx@hotmail.com Información artículo: Original - Cáncer testicular Trabajo recibido: octubre 2008

Trabajo aceptado: noviembre 2008 